\title{
Recent Advances in Inhibited-Coupling Guiding Hollow-Core Optical Fibers
}

\author{
Jonas H. Osório ${ }^{1}$, Foued Amrani ${ }^{1}$, Frédéric Delahaye ${ }^{1}$, Matthieu Chafer ${ }^{1,2}$, Martin Maurel ${ }^{1,2}$, Christine \\ Restoin $^{1}$, Jean-Marc Blondy ${ }^{1}$, Benoît Debord ${ }^{1,2}$, Frédéric Gérôme ${ }^{1,2}$, Fetah Benabid ${ }^{1,2}$ \\ ${ }^{1}$ GPPMM Group, XLIM Research Institute, CNRS UMR 7252, University of Limoges, Limoges, France \\ ${ }^{2}$ GLOphotonics, Ester Technopôle, Limoges, France \\ e-mail:f.benabid@xlim.fr
}

\begin{abstract}
In this talk, we report on recent developments on inhibited-coupling guiding hollow-core optical fibers ranging from the understanding of the guidance mechanism to application fields. We show that a deep understanding of the cladding properties of those fibers allow to obtain fibers with optimized properties. In particular, we demonstrate that, by adequately designing and controlling the fiber physical parameters, one can obtain ultralow loss fibers for the ultraviolet, visible and infrared ranges. Additionally, we show that convenient alterations in the fiber cladding can modify the fiber mode loss hierarchy allowing it to act as a mode shaper, and polarization discriminator.
\end{abstract}

Keywords: fiber optics, hollow-core fibers, photonic-crystal fibers

\section{INTRODUCTION}

The field of hollow-core photonic-crystal fibers (HC-PCFs) has experienced very important progress in the latest years. This development was observed on both the understanding of the light guidance mechanisms and on the application of these fibers in several practical areas in photonics. In this framework, the research on the HC-PCFs guidance properties allowed to identify the photonic bandgap (PBG) [1] and the inhibited-coupling (IC) [2] as the guiding mechanisms which govern light transmission in $\mathrm{HC}-\mathrm{PCF}$.

In PBG fibers, the cladding effective index-frequency space is structured in regions which correspond to the cladding mode bands and to the gaps in which no cladding mode can exist. Thus, light guidance in PBG fibers occur thanks to the inexistence of cladding modes in the core-guided mode effective index-frequency space to which the core mode could be coupled to.

Contrariwise, the cladding effective index-frequency space of IC fibers presents no gaps and, therefore, core and cladding modes coexist. In this context, light guidance in IC fibers relies on the minimization of the coupling between the core and cladding modes fields. This condition is represented by $\left\langle\phi_{\text {clad }}\left|\Delta n^{2}\right| \phi_{\text {core }}\right\rangle \rightarrow 0$, where $\left|\phi_{\text {core }}\right\rangle$ and $\left|\phi_{\text {clad }}\right\rangle$ are, respectively, the core and cladding modes fields and $\Delta n$ is a transverse index profile function $[3,4]$. The minimization of the coupling of core and cladding modes can be accomplished either by reducing the spatial overlap between the core and cladding mode fields or by attaining a strong mismatch between these modes transverse spatial phases [3, 4].

The minimum loss figure measured for PBG fibers is $1.2 \mathrm{~dB} / \mathrm{km}$ around $1600 \mathrm{~nm}$ [5]. This value is one order of magnitude above the minimum value obtained for solid-core fibers in the same wavelength range, $0.14 \mathrm{~dB} / \mathrm{km}$ [6]. For shorter wavelengths, the lowest reported loss figure in PBG fibers was $870 \mathrm{~dB} / \mathrm{km}$ at $557 \mathrm{~nm}$ [7]. Reducing the attenuation values in PBG fibers, however, is highly challenging due to the existence of limiting factors such as the strong core-cladding optical overlap, the presence of interface surface modes and the core surface roughness. Additionally, PBG fibers guiding in short wavelengths $(<1 \mu \mathrm{m})$ depend upon smaller cladding pitches and core diameters, which introduce additional challenges in their fabrication process.

Conversely, IC guiding HC-PCFs has core-cladding optical overlap with several orders of magnitude lower that PBG guiding HC-PCFs and are able to guide light in the short wavelength range with much larger core and cladding sizes. Thus, they appear as the sole candidates able to overcome PBG fibers disadvantages. A crucial step towards loss reduction in IC fibers was the introduction of the hypocycloid core contour (negative curvature) [8, 9]. In fact, the use of the hypocycloid core contour implied on a remarkable reduction of the attenuation values in IC fibers from the decibel per meter $(\mathrm{dB} / \mathrm{m})$ level in the first kagomé-lattice fiber [10] to the recently demonstrated loss of $8.5 \mathrm{~dB} / \mathrm{km}$ at $1030 \mathrm{~nm}$ [11]. For shorter wavelengths, however, the loss of kagomé-lattice fibers remain at the level of $70 \mathrm{~dB} / \mathrm{km}$ at $600 \mathrm{~nm}$ [13].

A specific kind of IC fibers which appeared as an extension of the hypocycloid core contour concept are the single-ring tubular lattice (SR-TL) HC-PCFs [14]. The cladding microstructure of the SR-TL HC-PCFs is endowed with a single ring of tubes with demarks a hypocycloid-shaped core without any connecting node. As both the hypocycloid core contour and the absence of connecting nodes in the fiber cladding favor IC guidance, notable results have been accomplished with this kind of fibers regarding the reduction of transmission loss values.

In this context, Debord et al. performed a systematic experimental and numerical study on the design of low loss SR-TL HC-PCF having demonstrated a loss figure of $7.7 \mathrm{~dB} / \mathrm{km}$ at $780 \mathrm{~nm}$ for a SR-TL HC-PCF with a cladding formed of 8 tubes [3]. Moreover, modified designs of tubular lattice HC-PCFs using so-called 
conjoined [15] and nested tubes [16] have been reported to reach loss values of $2 \mathrm{~dB} / \mathrm{km}$ at $1512 \mathrm{~nm}$ and $1.3 \mathrm{~dB} / \mathrm{km}$ at $1450 \mathrm{~nm}$, respectively. For shorter wavelengths, in turn, the loss of SR-TL HC-PCF are reported to be as low as $80 \mathrm{~dB} / \mathrm{km}$ at $532 \mathrm{~nm}$ [17], $130 \mathrm{~dB} / \mathrm{km}$ at $300 \mathrm{~nm}$ [18], and $100 \mathrm{~dB} / \mathrm{km}$ at $218 \mathrm{~nm} \mathrm{[19].} \mathrm{The} \mathrm{recent} \mathrm{results} \mathrm{on} \mathrm{the} \mathrm{IC}$ fibers show that, for wavelengths lower than $1 \mu \mathrm{m}$, the attenuation values are no longer limited by the fiber design but by the surface scattering loss (SSL) which results from the core surface roughness which arises from capillary waves or instabilities during the fiber drawing [3].

In this paper, we report on the recent advances on IC guiding HC-PCFs. In particular, we report on a SR-TL HC-PCF with a 9 tubes cladding structure with record loss of $13.8 \mathrm{~dB} / \mathrm{km}$ at $539 \mathrm{~nm}$ and which was drawn over $1 \mathrm{~km}$ with very high outer diameter (OD) stability. Moreover, we show that convenient modification in the fiber cladding can modify the fiber mode loss hierarchy allowing it to act as a mode shaper, and polarization discriminator.

\section{RECORD LOW LOSS SR-TL HC-PCF IN THE GREEN SPECTRAL RANGE}

In this section, we report on a SR-TL HC-PCF with record low loss in the green spectral range. Figure 1a presents the cross-section of the fiber we describe herein. The fiber cladding is composed by a single ring of 9 tubes, which establishes a $42 \mu \mathrm{m}$ core diameter. The thickness of the cladding tubes is $610 \mathrm{~nm}$ and the distance between the lattice tubes range from $2.2 \mu \mathrm{m}$ to $4.0 \mu \mathrm{m}$. Here, we employ a 9 tubes lattice fiber having into account the results of [3], which demonstrated that, for the same core diameter, the fiber confinement loss (CL) decreases with increasing number of lattice tubes. Additionally, the use of 9 tubes in the fiber cladding allows to maintain a good compromise between low CL and a modal content dominated by the fundamental mode.

Figure $1 \mathrm{~b}$ presents the fiber OD evolution during the fiber draw. It is seen that the fiber was drawn over $1 \mathrm{~km}$ with very high OD stability. By fitting a gaussian function to the experimental data, we obtained a $2 \sigma$ value of $0.44 \mu \mathrm{m}$, which means a $0.2 \%$ variation in the OD values. The $0.2 \%$ OD variation is indicated as the blue dashed lines in Figure 1b.

The fiber loss was measured in several cutback measurements, which took into account the transmission spectra for the fiber lengths of $1 \mathrm{~km}, 500 \mathrm{~m}, 200 \mathrm{~m}$ and $5 \mathrm{~m}$. Figure 1c presents the average loss of the fiber when considering all the cutback measurements. The average minimum loss figures were measured as $90 \mathrm{~dB} / \mathrm{km}$ at $1700 \mathrm{~nm}, 11.4 \mathrm{~dB} / \mathrm{km}$ at $850 \mathrm{~nm}$ and $16 \mathrm{~dB} / \mathrm{km}$ at $550 \mathrm{~nm}$. Moreover, cutback measurements were performed by using lasers at $343 \mathrm{~nm}$ and $355 \mathrm{~nm}$. At these wavelengths, loss values of $150 \mathrm{~dB} / \mathrm{km}$ and $75 \mathrm{~dB} / \mathrm{km}$ were measured, respectively (green dots in Figure 1c; the transmission spectra, T, is also shown in Figure 1c for better visualization of the loss figures at $343 \mathrm{~nm}$ and $355 \mathrm{~nm}$ within the fiber transmission band). It is worth saying that the measured loss value at $355 \mathrm{~nm}$ is lower than the figures reported in [17] and [18] for similar wavelengths.

Figure 1d presents a close-up of the measured loss in the wavelength range between $400 \mathrm{~nm}$ and $650 \mathrm{~nm}$ for the cutback measurements performed between the fiber lengths of $1 \mathrm{~km}$ and $5 \mathrm{~m}, 500 \mathrm{~m}$ and $5 \mathrm{~m}$, and $200 \mathrm{~m}$ and $5 \mathrm{~m}$. It is observed that the loss values are lower for the cutback measurements using shorter fiber lengths. For instance, at $539 \mathrm{~nm}$, the accounted loss values were $19 \mathrm{~dB} / \mathrm{km}$ for the measurement using the $1 \mathrm{~km}$ and $5 \mathrm{~m}$ long pieces, $17.3 \mathrm{~dB} / \mathrm{km}$ for the cutback between the $500 \mathrm{~m}$ and $5 \mathrm{~m}$ long fibers, and $13.8 \mathrm{~dB} / \mathrm{km}$ for the measurement performed with the $200 \mathrm{~m}$ and $5 \mathrm{~m}$ long pieces. The discrepancy between the loss figures is attributed to possible structural non-uniformities along the fiber length. It is noteworthy that the $13.8 \mathrm{~dB} / \mathrm{km}$ attenuation value at $539 \mathrm{~nm}$ is as low as the Rayleigh scattering limit of bulk silica (green curve in Figure 1d) at this wavelength. This loss
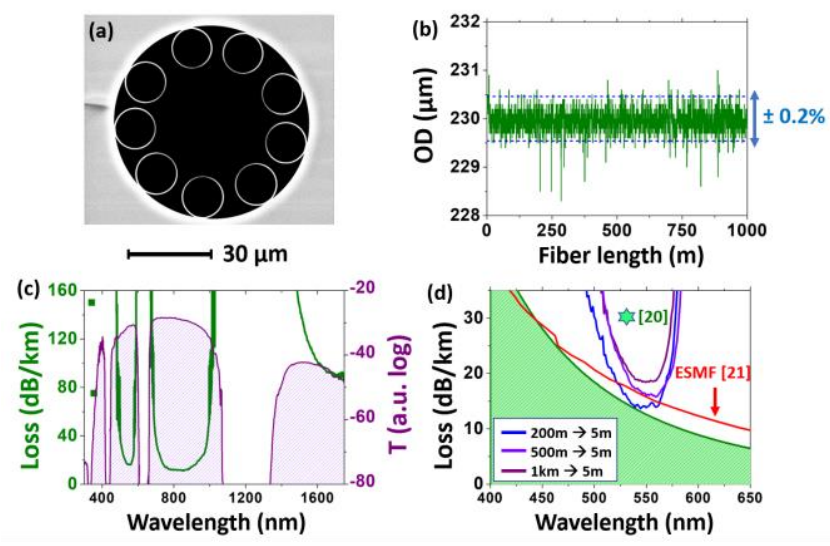

Figure 1. (a) Fiber cross-section and (b) fiber outer diameter (OD) evolution during the fiber fabrication. (c) Average loss considering cutback measurements between the fiber lengths of $1 \mathrm{~km}, 500 \mathrm{~m}, 200 \mathrm{~m}$ and $5 \mathrm{~m}$ and fiber transmission spectrum ( $T$; right axis). (d) Measured loss considering the $1 \mathrm{~km}$ and $5 \mathrm{~m}, 500 \mathrm{~m}$ and

$5 \mathrm{~m}, 200 \mathrm{~m}$ and $5 \mathrm{~m}$ fiber pieces. The red line presents the loss of an endlessly single-mode fiber (ESMF)

[21] and the star symbol the loss of a single mode fiber optimized for the visible spectral range [20]. 
value is lower than any value reported for other fibers in the spectral range between $520 \mathrm{~nm}$ and $560 \mathrm{~nm}$, including commercial fibers which were optimized to work in the visible wavelength range (whose attenuation figures are around 20-30 dB/km) [19-21]. Figure 1d presents the loss values for an endlessly single-mode fiber (ESMF, red line in Figure 1d) [21] and the attenuation for a solid-core single-mode fiber optimized for the visible wavelength range [20], used here for comparison. Further information on this research can be found in [22].

\section{CORE MODAL STRUCTURING IN IC GUIDING FIBERS}

Deep knowledge of the cladding properties in IC fibers is crucial for successfully achieving the desired properties and performances in these fibers. In the last section, we showed that an adequate design of the fiber cladding allows to attain ultralow loss in these fibers. Additionally, we present in this section that a convenient alteration of the cladding tubes azimuthal position allows modifying the mode loss hierarchy in IC fibers, which can be employed for favoring the propagation of specific high order modes (HOMs). The concept is based on adequately enlarging the spacing between selected lattice tubes for providing additional leakage to the fundamental mode while keeping low influence on the CL of specific HOMs. This property allows to obtain unusual intensity and polarization profiles at the fiber output. The fiber can, therefore, act as a mode shaper.

Figure $2 \mathrm{a}$ presents three fiber designs (FDs) we study herein. Additionally, it is shown in Figure 2a the simulated CL of the fundamental mode and of selected HOMs for each FD. FD\#1 consists of a 10-tubes lattice fiber. As expected, in this FD, the lowest loss mode is the fundamental one ( $3.6 \mathrm{~dB} / \mathrm{km}$ at $1000 \mathrm{~nm})$. In FD\#2, the creation of two larger gap between the lattice tubes (oriented at $180^{\circ}$ ) causes the $\mathrm{CL}$ of the $\mathrm{LP}_{01}$ and $\mathrm{LP}_{11 \mathrm{~b}}$ modes to increase by two orders of magnitude. In contrast, this alteration in the cladding tubes distribution has low impact on the $\mathrm{LP}_{11 \mathrm{a}}$ mode CL (modal profiles in Figure 2a), causing this mode to be the lowest loss one in FD\#2 (33 dB/km at $1000 \mathrm{~nm}$ ). In FD\#3, in turn, four larger gaps oriented at $90^{\circ}$ are added to the fiber cladding structure. This modification allows favoring the propagation of $\mathrm{LP}_{21}$ mode $(80 \mathrm{~dB} / \mathrm{km}$ at $1000 \mathrm{~nm})$.

Additionally, in Figure 2a, Poynting vector $\left(\mathrm{p}_{\mathrm{r}}\right)$ plots are presented for mapping the channels of power leakage in the studied FDs. For example, it is seen that, in FD\#2, the enlargement of the distance between the lattice tubes at $180^{\circ}$ causes the spacing between the cladding tubes to be the main leakage channel for $\mathrm{LP}_{01}$ and $\mathrm{LP}_{11 \mathrm{~b}}$ modes. As a consequence of that, the $\mathrm{CL}$ values for the $\mathrm{LP}_{01}$ and $\mathrm{LP}_{11 \mathrm{~b}}$ significantly increases in comparison to their $\mathrm{CL}$ in FD\#1. In contrast, the addition of larger gaps at $180^{\circ}$ in the cladding structure have low impact on the $\mathrm{p}_{\mathrm{r}}$ distribution for the $\mathrm{LP}_{11 \mathrm{a}}$ mode. It causes the influence on the CL of $\mathrm{LP}_{11 \mathrm{a}}$ mode to be much weaker.

Two fibers (F\#1 and F\#2) were drawn and the loss of selected HOM were measured. The cutback measurement results and the near field profiles at the fiber output are shown in Figure 2b. At $1000 \mathrm{~nm}$, loss values around $200 \mathrm{~dB} / \mathrm{km}$ and $400 \mathrm{~dB} / \mathrm{km}$ could be accounted for the $\mathrm{LP}_{11}$ mode in F\#1 and for the $\mathrm{LP}_{21}$ mode in F\#2, respectively. Lastly, mode transformations tests were performed by using F\#1 and F\#2. Figure 2c shows the unusual fiber output profiles that could be obtained by conveniently coupling a superposition of $L_{01}$ and $L P_{11}$ modes in F\#1 and by altering the polarization of the input light. Particularly, the lobes in the F\#1 output LP 11 -like profile has orthogonal polarization states, which is noteworthy. We envisage that this novel concept will find applications atom optics, atom-surface physics, sensing and nonlinear optics experiments. Further information on this research can be found in [23].
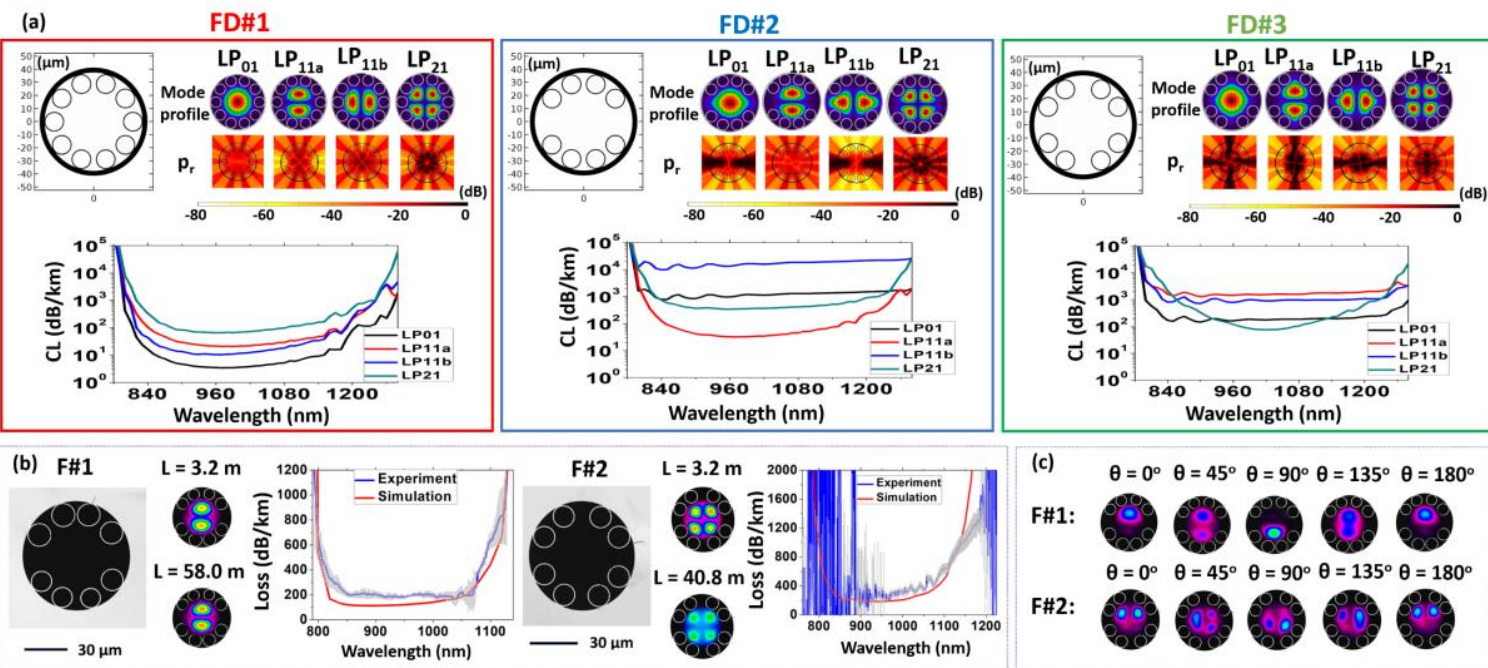

Figure 2. (a) Fiber designs (FDs), mode profiles, Poynting vector plots $\left(p_{r}\right)$ and the CL accounted from simulations. (b) Fabricated fibers (F\#1 and F\#2) and loss estimation of HOMs. (c) Output of F\#1 and F\#2 for different input polarization angles. 


\section{CONCLUSIONS}

In this manuscript, we described recent advances on IC guiding HC-PCFs. In this context, we reported on a SRTL HC-PCF with a 9 tubes cladding structure with record loss of $13.8 \mathrm{~dB} / \mathrm{km}$ at $539 \mathrm{~nm}$ and which was drawn over $1 \mathrm{~km}$ with very high OD stability. Additionally, we presented that a suitable modification of the fiber cladding tubes arrangement can alter the fiber mode loss hierarchy. It allows the fiber to act as a mode shaper and polarization discriminator.

\section{ACKNOWLEDGEMENTS}

This research was funded through grants $\sum$ LIM Labex Chaire, PIA 4F project and the région of Nouvelle Aquitaine.

\section{REFERENCES}

[1] T. A. Birks et al. "Full 2-D photonic bandgaps in silica/air structures," Electron. Lett., 31, 22, 1995.

[2] F. Couny et al. "Generation and photonic guidance of multi-octave optical-frequency combs," Science, 318, 1118-1121, 2007.

[3] B. Debord et al. "Ultralow transmission loss in inhibited-coupling guiding hollow fibers," Optica, 4, 209217, 2017.

[4] B. Debord et al. "Hollow-core fiber technology: the rising of "gas photonics", Fibers, 7(2), 16, 2019.

[5] P. J. Roberts et al. "Ultimate low loss of hollow-core photonic crystal fibers," Optics Express, 13, 1, 236244, 2005.

[6] Y. Tamura et al., "The first 0.14-dB/km loss optical fiber and its impact on submarine transmission," Journal of Lightwave Technology, 36, 44-49, 2018.

[7] P. J. Mosley et al. "Ultrashort pulse compression and delivery in a hollow-core photonic crystal fiber at 540 nm wavelength," Optics Letters, 35, 3589-3591, 2010.

[8] Y. Wang et al. "Low loss broadband transmission in optimized core-shape Kagome hollow-core PCF," in Conference on Lasers and Electro-Optics (OSA, 2010), paper CPDB4.

[9] Y. Y. Wang et al. "Low loss broadband transmission in hypocycloid-core Kagome hollow-core photonic crystal fiber," Optics Letters, 36, 669-671, 2011.

[10] F. Benabid et al. "Stimulated Raman scattering in hydrogen-filled hollow-core photonic crystal fiber," Science, 298, 5592, 399-402, 2002.

[11] M. Maurel et al. "Optimized inhibited-coupling Kagome fibers at Yb-Nd:Yag (8.5 dB/km) and Ti:Sa (30 $\mathrm{dB} / \mathrm{km}$ ) ranges," Optics Letters, 43, 1598-1601, 2018.

[12] B. Debord et al. "Ultra low-loss hypocycloid-core Kagome hollow-core photonic crystal fiber for green spectral-range applications," Optics Letters, 39, 6245-6248, 2014.

[13] A. D. Pryamikov et al. "Demonstration of a waveguide regime for a silica hollow-core microstructured optical fiber with a negative curvature of the core boundary in the spectral region $>3.5 \mu \mathrm{m}$," Optics Express, 19, 1441-1448 (2011).

[14] S. Gao et al. "Hollow-core conjoined-tube negative-curvature fibre with ultralow loss," Nature Communications, 9, 2828, 2018.

[15] T. D. Bradley et al. "Record low-loss $1.3 \mathrm{~dB} / \mathrm{km}$ data transmitting antiresonant hollow fibre," in European Conference on Optical Communication, 2018.

[16] S. Gao et al. "Nodeless hollow-core fiber for the visible spectral range," Optics Letters, 42, 61-64, 2017.

[17] S. Gao et al. "Hollow-core negative-curvature fiber for UV guidance," Optics Letters, 43, 1347-1350, 2018.

[18] F. Yu et al. "Single-mode solarization-free hollow-core fiber for ultraviolet pulse delivery," Optics Express, 26, 10879-10887, 2018.

[19] Thorlabs, Single Mode Fiber: 400 to $680 \mathrm{~nm}$, S405-XP. https://www.thorlabs.com/drawings/360e4ff3e1d0d7eb-98693A0E-05E3-90D71DEBC16BFC84B4B7/S405-XP-SpecSheet.pdf

[20] Corning RGB 400 Specialty Optical Fiber. https://www.corning.com/media/worldwide/global/documents/sfiber\%20RGB\% $20400 \% 20$ Specialty\%20Fi ber\%20PDF.pdf

[21] Thorlabs, Endlessy Single Mode, Large-Mode-Area-Fiber, https://www.thorlabs.com/newgrouppage9.cfm?objectgroup_id=1902.

[22] M. Chafer et al. " $1 \mathrm{~km}$ hollow-core fiber with loss at the silica Rayleigh limit in the green spectral region," IEEE Photonics Technology Letters, 31, 685-688, 2019.

[23] J. H. Osório et al. "Tailoring modal properties of inhibited-coupling fibers by cladding modification," Scientific Reports, 9:1376, 2019. 